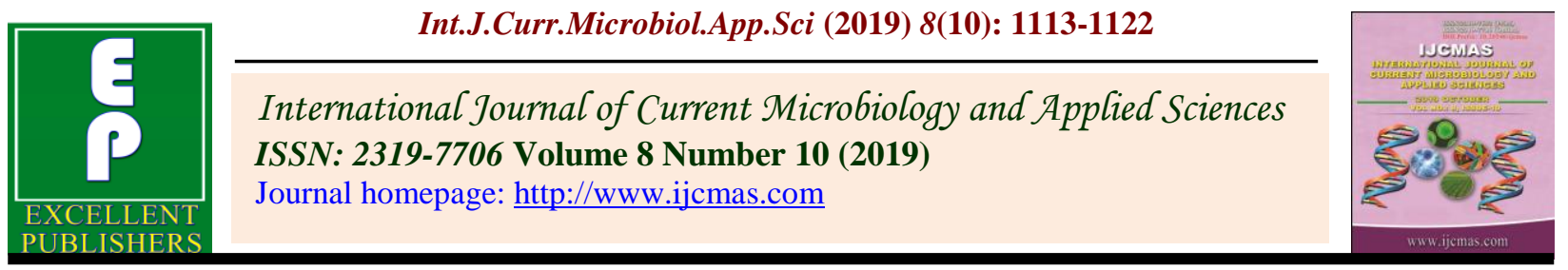

Original Research Article

https://doi.org/10.20546/ijcmas.2019.810.130

\title{
Effect of Plant Defense Activators on White Rust of Mustard CV. RH- 749 under Artificial Epiphytotic Conditions
}

\author{
Hanuman Singh $^{1 *}$, R.S. Ratnoo ${ }^{2}$, A. Trivedi ${ }^{2}$, H.K. Jain ${ }^{2}$, V. Saharan ${ }^{2}$ and F.L. Sharma ${ }^{2}$ \\ ${ }^{1}$ College of Horticulture and Forestry, Agriculture University, Kota, India \\ ${ }^{2}$ Maharana Pratap University of Agriculture and Technology, Udaipur, India \\ *Corresponding author
}

\section{A B S T R A C T}

\section{Keywords}

Plant defense activators,

Mustard,

Epiphytotic

\section{Article Info}

Accepted:

10 September 2019

Available Online:

10 October 2019
The size of pustule of white rust was recorded minimum in Metalaxyl $0.3 \%$ was $0.32 \mathrm{~mm}$ followed by Metalaxyl $0.2 \%(0.33 \mathrm{~mm})$ in cv. RH-749 during 2015-16 while maximum size of pustules were observed in check was $6.55 \mathrm{~mm}$ followed by zinc sulphate at $0.50 \%$ (5.88 mm) during 2016-17. Among the abiotic chemicals, salicylic acid was recorded significantly better over all the treatments. The number of pustules were recorded maximum in the check was 7.47 and 7.53 followed by zinc sulphate at $0.50 \%$ was 7.13 and 7.27 during 2015-16 and 2016-17, respectively. Salicylic acid $0.25 \%$ reduce $31.50 \%$ white rust disease over the control followed by calcium sulphate at $1.00 \%$ which reduce disease $23.99 \%$. Zinc sulphate at $0.50 \%$ was found least effective abiotic chemical which reduce $6.14 \%$ disease.

\section{Introduction}

Plants undergo several stresses throughout their life span and respond them through numerous chemical and physical stimuli (Omidi, 2010). Plants are prepared through pre-formed or constitutive mechanical and chemical barriers as well as with inducible defense systems to protect themselves from the attack of different pathogens such as bacteria, virus, fungi, nematodes and parasitic plants in their environment. Induction of defensive system in plants can be achieved or enhanced by pretreatment of plants with avirulent pathogen (biotic inducers) or chemical compounds (abiotic inducers). These biotic and abiotic inducers are known as elicitors. Elicitors are low molecular weight compounds which induces a systemic defense response in plants. These elicitors are synthesized by itself or released from polymeric precursors through infection by pathogen (Ozeretskovskaya and Vasyukova, 2002; Zhao et al., 2005, Holopainen et al., 2009).

Induced resistance has been exploited extensively for the management of many plant diseases (Gorlach et al., 1996). Several compounds such as salicylic acid (Yalpani and Raskin, 1993), benzothiadiazoles (Narusaka, et al., 1999), fatty acids and oligosaccharides 
(Kobayashi, et al., 1993), harpin protein (Wei et al., 1992, De et al., 1990) are effective inducers of plant defense in the host plant system. In addition, numerous microorganisms applied to the leaves or roots of plants may induce systemic or local resistance (Liu, et al., 1995). Such resistance is reported to be active against several types of organisms such as fungi, bacteria and even parasitic plants (Matheron and Porchas, 2002).

Recent years, a new group of compounds that activate host defense mechanism and protect the plant against pathogens has been developed to manage crop diseases. These chemicals are known as "plant defense activators" or "plant activators" (Romero et al., 2001). Salicylic acid mimics compounds such as acibenzolar s methyl, bion, phosphorous salts and micronutrient potassium salts have been used as commercial plant activators (Becot et al., 2000; Macmillan et al., 2000; Pajot et al., 2001; Graham and Leite, 2004).

Biological plant defense inducers such as Trichoderma viride, Pseudomonas, Bacillus, Serratia, nonpathogenic strains of Fusarium and yeast have been developed as commercial product to combat various plant diseases (Droly et al., 2002; Benhamon and Garand, 2001; Verhagen et al., 2004; Howell et al., 2000). Trichoderma viride is known as one of the most important bio-control agent that has been used extensively in agriculture. Biological plant defense inducers provide systemic resistance to plants infested by various fungal and bacterial phytopathogens. Biocontrol activity of Trichoderma based biocontrol agents inheres in their ability to orchestrate several biochemical pathways in diseased plants (Surekha et al., 2014). Although studies explaining biocontrol activity of Trichoderma against fungal pathogens are recognized, there is need for imparting the biochemical basis of disease resistance being induced by Trichoderma. Therefore, research investigations pertaining to induction of such systemic resistance and associated biochemical responses is necessary to understand the mechanism of biological control activity of Trichoderma viride and Pseudomonas fluorescens.

Host plant defense can be induced by the application of non-pathogenic microorganisms (Vishwanath et al., 1999; Singh et al., 1999) and certain abiotic activators such as salicylic acid (Spletzer and Enyedi, 1999) and amino butyric acid (Cohen, 1994; Kaur and Kolte, 2001). Host resistance is considered to be the most effective and economical method in plant disease management. Evaluation of new germplasm helps to identify some good sources of resistance that may be exploited for commercial production or for breeding of new disease resistant lines. The resistant sources help to slow down the rate of epidemic buildup. Therefore, the development of disease resistant varieties is the most effective and economical approach of integrated disease management programme.

In plants systemic resistant can be induced through biotic or abiotic plant defense inducers. Resistance to diseases can be induced systematically in plants by biological and chemical means (Ryals et al., 1994; Spletzer and Enyedi, 1999). Most commonly used chemicals inducers are salicylic acid, which appears to mimic the systemic effects of localized infection in plant system (Safari et al., 2013). One of the potential management methods is the use of systemic acquired resistance to trigger host defense mechanisms, which would not involve the application of hazardous compounds to plants (Durrant and Dong, 2004). External application of salicylic acid can induce systemic acquired resistance (Hammerschmidt, 1999).

The potential of chemical inducers of systemic acquired resistance to reduce white rust 
disease on mustard was evaluated to explore the possibility of utilizing induced host resistance as a realistic alternative to classical fungicides in disease management.

\section{Materials and Methods}

First spray plant defense activators in the mustard variety RH-749 when plants shows first symptom of the disease in each treatment with their respective concentration using a randomized block design. One standard chemical check Metalaxyl at 0.10, 0.20 and 0.30 per cent and one sterile distilled water check were also maintained in three replications.

\section{Observations recorded}

\section{Size of pustule on leaves}

Diameter of randomly selected five leaves was measured in mm with the help of plastic scale and average size of pustule was calculated and recorded at ten days interval.

\section{Number of pustules}

Numbers of pustules recorded by counting the pustules per $25 \mathrm{~mm}^{2}$ leaf area of randomly selected five leaves of plant. The observations were recorded on five leaves and average number of pustules was then calculated per 25 $\mathrm{mm}^{2}$ leaf area.

\section{Per cent disease index on leaf}

The per cent disease index on leaf due to white rust was recorded at 10 days interval up to 90 days after sowing (DAS) by using of 0-5 rating scale given by Biswas et al., 2011; Tirmali and Kolte (2012). Ratings were given as per above mentioned rating scale and white rust per cent disease index was calculated by using formula given by Wheeler (1969) and Mathur et al., (2013). Observations were recorded by randomly selecting twenty five leaves from each replication and were rated as per the above rating scale and per cent disease index was calculated and statistically analyzed as described by Panse and Sukhatme (1985) for analysis of variance of randomized block design in order to test the significance of experimental results.

White rust index $(\%)=\frac{\text { Sum of all numerical ratings }}{\text { Number of leaves examined } X \text { maximum grade of scale }} \times 100$

\section{Results and Discussion}

The size of pustule was recorded minimum in Metalaxyl $0.3 \%$ was $0.32 \mathrm{~mm}$ followed by Metalaxyl $0.2 \%(0.33 \mathrm{~mm})$ in cv. RH-749 during 2015-16 (Table 2). However the different concentrations of Metalaxyl were found at par with each other during 2015-16 at 70,80 and 90 days after sowing of the crop. All the treatments were found significantly better in the comparison with the control. The maximum size of pustules was observed in check was $6.55 \mathrm{~mm}$ followed by zinc sulphate at $0.50 \%(5.88 \mathrm{~mm})$ during 2016-17. Among the abiotic chemicals, salicylic acid was recorded significantly better over all the treatments. Both the biotic agents were found significantly better in comparison with the control. The size of the pustules was enlarged from 70 to 90 days after sowing the mustard.

The number of pustules were recorded maximum in the check was 7.47 and 7.53 followed by zinc sulphate at $0.50 \%$ was 7.13 and 7.27 during 2015-16 and 2016-17, respectively (Table 3 ). The minimum number of pustules was found in Metalaxyl $0.2 \%$ and Metalaxyl $0.3 \%$ was 1.80 . However, different concentrations of Metalaxyl were found at par in comparison with each other during 2015-16 and 2016-17 at 70, 80 and 90 days after sowing. Among the biotic agents, 
Trichoderma viride and Pseudomonas fluorescens were found significantly better over the check except Trichoderma viride at 1:00 \% was found at par at 80 days after sowing during 2016-17. In non-conventional chemicals, salicylic acid was found superior followed by calcium sulphate then others which reduce average number of pustules $37.95 \%$ and $32.07 \%$ in comparison with the control, respectively and minimum reduction in number of pustules were recorded in zinc sulphate at $0.50 \%(7.40 \%)$ followed by Borax $0.50 \%(15.75 \%)$ (Table 1).

Table.1 Biotic and abiotic activators and their concentration under field study

\begin{tabular}{|l|l|c|}
\hline Treatments & Biotic and abiotic agents & Concentration (\%) \\
\hline $\mathbf{T}_{\mathbf{1}}$ & Trichoderma viride & 1.00 \\
\hline $\mathbf{T}_{\mathbf{2}}$ & Pseudomonas fluorescens & 1.00 \\
\hline $\mathbf{T}_{\mathbf{3}}$ & Salicylic acid & 0.25 \\
\hline $\mathbf{T}_{\mathbf{4}}$ & Borax $\left(\mathrm{Na}_{2} \mathrm{~B}_{4} \mathrm{O}_{7} \cdot 10 \mathrm{H}_{2} \mathrm{O}\right)$ & 0.50 \\
\hline $\mathbf{T}_{\mathbf{5}}$ & Potassium sulphate $\left(\mathrm{K}_{2} \mathrm{SO}_{4}\right)$ & 1.00 \\
\hline $\mathbf{T}_{\mathbf{6}}$ & Calcium sulphate $\left(\mathrm{CaSO}_{4}\right)$ & 1.00 \\
\hline $\mathbf{T}_{\mathbf{7}}$ & Metalaxyl & 0.10 \\
\hline $\mathbf{T}_{\mathbf{8}}$ & Metalaxyl & 0.20 \\
\hline $\mathbf{T}_{\mathbf{9}}$ & Metalaxyl & 0.30 \\
\hline $\mathbf{T}_{\mathbf{1 0}}$ & Potassium chloride $(\mathrm{KCl})$ & 1.00 \\
\hline $\mathbf{T}_{\mathbf{1 1}}$ & Zinc sulphate $\left(\mathrm{ZnSO} 4.7 \mathrm{H}_{2} \mathrm{O}\right)$ & 0.50 \\
\hline $\mathbf{T}_{\mathbf{1 2}}$ & Check & - \\
\hline
\end{tabular}

\begin{tabular}{|c|c|}
\hline Numerical rating & Leaf area covered by the pustules (\%) \\
\hline $\mathbf{0}$ & No symptoms \\
\hline $\mathbf{1}$ & $1-10$ \\
\hline $\mathbf{2}$ & $11-25$ \\
\hline $\mathbf{3}$ & $26-50$ \\
\hline $\mathbf{4}$ & $51-75$ \\
\hline $\mathbf{5}$ & $>75$ \\
\hline
\end{tabular}


Table.2 Effect of biotic and non-conventional chemicals on size of pustule on mustard cv. RH-749 under field conditions

\begin{tabular}{|c|c|c|c|c|c|c|c|c|c|c|c|}
\hline \multirow{3}{*}{ Treatment } & \multirow{3}{*}{$\begin{array}{c}\text { Concentration } \\
(\%)\end{array}$} & \multicolumn{10}{|c|}{ Size of pustules (mm) } \\
\hline & & \multicolumn{4}{|c|}{ 2015-16 } & \multicolumn{4}{|c|}{ 2016-17 } & \multirow{2}{*}{$\begin{array}{c}\text { Over all } \\
\text { Mean }\end{array}$} & \multirow{2}{*}{$\begin{array}{l}\text { Reduction ove } \\
\text { check }(\%)\end{array}$} \\
\hline & & 70 DAS & 80 DAS & 90 DAS & Mean & 70 DAS & 80 DAS & 90 DAS & Mean & & \\
\hline $\begin{array}{c}\text { Trichoderma } \\
\text { viride }\end{array}$ & 1.00 & 0.90 & 3.03 & 5.51 & 3.15 & 1.02 & 3.05 & 5.61 & 3.23 & 3.19 & 19.90 \\
\hline $\begin{array}{l}\text { Pseudomonas } \\
\text { fluorescens }\end{array}$ & 1.00 & 0.57 & 2.23 & 4.72 & 2.51 & 0.67 & 2.27 & 4.81 & 2.59 & 2.55 & 35.98 \\
\hline Salicylic Acid & 0.25 & 0.43 & 1.84 & 4.01 & 2.09 & 0.53 & 1.84 & 4.08 & 2.15 & 2.12 & 46.73 \\
\hline Borax & 0.50 & 0.82 & 2.63 & 5.19 & 2.88 & 0.89 & 2.70 & 5.28 & 2.96 & 2.92 & 26.66 \\
\hline $\mathrm{K}_{2} \mathrm{SO}_{4}$ & 1.00 & 0.64 & 2.51 & 4.98 & 2.71 & 0.74 & 2.59 & 5.08 & 2.80 & 2.76 & 30.76 \\
\hline $\mathrm{CaSO}_{4}$ & 1.00 & 0.49 & 2.10 & 4.39 & 2.32 & 0.56 & 2.17 & 4.48 & 2.40 & 2.36 & 40.62 \\
\hline Metalaxyl 0.1\% & 0.10 & 0.35 & 1.64 & 3.40 & 1.80 & 0.43 & 1.71 & 3.48 & 1.87 & 1.83 & 53.93 \\
\hline Metalaxyl $0.2 \%$ & 0.20 & 0.33 & 1.57 & 3.23 & 1.71 & 0.41 & 1.64 & 3.35 & 1.80 & 1.75 & 55.92 \\
\hline Metalaxyl $0.3 \%$ & 0.30 & 0.32 & 1.58 & 3.17 & 1.69 & 0.43 & 1.58 & 3.28 & 1.76 & 1.73 & 56.61 \\
\hline $\mathrm{KCl}$ & 1.00 & 0.51 & 2.15 & 4.49 & 2.38 & 0.60 & 2.25 & 4.64 & 2.50 & 2.44 & 38.69 \\
\hline $\mathrm{ZnSO}_{4}$ & 0.50 & 0.95 & 3.12 & 5.82 & 3.30 & 1.15 & 3.15 & 5.88 & 3.39 & 3.35 & 15.94 \\
\hline Check & - & 1.36 & 4.02 & 6.46 & 3.95 & 1.45 & 4.05 & 6.55 & 4.01 & 3.98 & 0.00 \\
\hline SEm \pm & - & 0.02 & 0.07 & 0.14 & - & 0.02 & 0.06 & 0.08 & - & - & - \\
\hline CD $5 \%$ & - & 0.05 & 0.21 & 0.41 & - & 0.05 & 0.17 & 0.23 & - & - & - \\
\hline
\end{tabular}

DAS $=$ Days after sowing 
Table.3 Effect of biotic and abiotic agents on number of white rust leaf pustule on mustard cv. RH-749 under field condition

\begin{tabular}{|c|c|c|c|c|c|c|c|c|c|c|c|}
\hline \multirow{3}{*}{ Treatment } & \multirow{3}{*}{$\begin{array}{c}\text { Concentration } \\
(\%)\end{array}$} & \multicolumn{10}{|c|}{ Number of pustules/25 $\mathrm{mm}^{2}$ area } \\
\hline & & \multicolumn{4}{|c|}{ 2015-16 } & \multicolumn{4}{|c|}{$2016-17$} & \multirow{2}{*}{$\begin{array}{l}\text { Over all } \\
\text { Mean }\end{array}$} & \multirow{2}{*}{$\begin{array}{l}\text { Reduction over } \\
\text { check }(\%)\end{array}$} \\
\hline & & 70 DAS & 80 DAS & 90 DAS & Mean & 70 DAS & 80 DAS & 90 DAS & Mean & & \\
\hline $\begin{array}{c}\text { Trichoderma } \\
\text { viride }\end{array}$ & 1.00 & 3.53 & 5.20 & 7.00 & 5.24 & 3.60 & 5.53 & 7.07 & 5.40 & 5.32 & 9.11 \\
\hline $\begin{array}{l}\text { Pseudomonas } \\
\text { fluorescens }\end{array}$ & 1.00 & 2.80 & 4.40 & 5.93 & 4.38 & 2.87 & 4.13 & 6.00 & 4.33 & 4.36 & 25.62 \\
\hline Salicylic Acid & 0.25 & 2.40 & 3.40 & 4.93 & 3.58 & 2.47 & 3.60 & 5.00 & 3.69 & 3.63 & 37.95 \\
\hline Borax & 0.50 & 3.20 & 4.87 & 6.60 & 4.89 & 3.47 & 4.80 & 6.67 & 4.98 & 4.93 & 15.75 \\
\hline $\mathrm{K}_{2} \mathrm{SO}_{4}$ & 1.00 & 3.13 & 4.67 & 6.20 & 4.67 & 3.20 & 4.93 & 6.27 & 4.80 & 4.73 & 19.17 \\
\hline $\mathrm{CaSO}_{4}$ & 1.00 & 2.67 & 3.80 & 5.33 & 3.93 & 2.73 & 3.93 & 5.40 & 4.02 & 3.98 & 32.07 \\
\hline Metalaxyl 0.1\% & 0.10 & 2.00 & 3.13 & 4.33 & 3.16 & 2.07 & 3.13 & 4.40 & 3.20 & 3.18 & 45.73 \\
\hline Metalaxyl $0.2 \%$ & 0.20 & 1.80 & 3.00 & 4.27 & 3.02 & 1.87 & 3.07 & 4.33 & 3.09 & 3.06 & 47.82 \\
\hline Metalaxyl $0.3 \%$ & 0.30 & 1.80 & 2.87 & 4.27 & 2.98 & 1.87 & 2.87 & 4.40 & 3.04 & 3.01 & 48.58 \\
\hline $\mathrm{KCl}$ & 1.00 & 2.93 & 4.00 & 5.47 & 4.13 & 3.00 & 4.07 & 5.53 & 4.20 & 4.17 & 28.84 \\
\hline $\mathrm{ZnSO}_{4}$ & 0.50 & 3.87 & 5.27 & 7.13 & 5.42 & 3.67 & 5.33 & 7.27 & 5.42 & 5.42 & 7.40 \\
\hline Check & - & 4.13 & 5.87 & 7.47 & 5.82 & 4.20 & 5.93 & 7.53 & 5.89 & 5.86 & 0.00 \\
\hline SEm \pm & - & 0.07 & 0.15 & 0.18 & - & 0.07 & 0.15 & 0.18 & - & - & - \\
\hline CD 5\% & - & 0.21 & 0.44 & 0.50 & - & 0.21 & 0.44 & 0.50 & - & - & - \\
\hline
\end{tabular}

DAS = Days after sowing 
Table.4 Effect of biotic and non-conventional chemicals on disease index of white rust on mustard cv. RH-749 under field conditions

\begin{tabular}{|c|c|c|c|c|c|c|c|c|c|c|c|}
\hline \multirow{3}{*}{ Treatment } & \multirow{3}{*}{$\begin{array}{c}\text { Concentration } \\
(\%)\end{array}$} & \multicolumn{10}{|c|}{ Disease index (\%) } \\
\hline & & \multicolumn{4}{|c|}{ 2015-16 } & \multicolumn{4}{|c|}{ 2016-17 } & \multirow{2}{*}{$\begin{array}{l}\text { Over all } \\
\text { Mean }\end{array}$} & \multirow{2}{*}{$\begin{array}{l}\text { Reduction ove } \\
\text { check }(\%)\end{array}$} \\
\hline & & 70 DAS & 80 DAS & 90 DAS & Mean & 70 DAS & 80 DAS & 90 DAS & Mean & & \\
\hline $\begin{array}{c}\text { Trichoderma } \\
\text { viride }\end{array}$ & 1.00 & $\begin{array}{c}24.27 \\
(29.51)\end{array}$ & $\begin{array}{c}42.67 \\
(40.78)\end{array}$ & $\begin{array}{c}76.53 \\
(61.03)\end{array}$ & $\begin{array}{c}47.82 \\
(43.75)\end{array}$ & $\begin{array}{c}25.07 \\
(30.04)\end{array}$ & $\begin{array}{c}42.93 \\
(40.94)\end{array}$ & $\begin{array}{c}77.33 \\
(61.57)\end{array}$ & $\begin{array}{c}48.44 \\
(44.11)\end{array}$ & 48.13 & 0.82 \\
\hline $\begin{array}{l}\text { Pseudomonas } \\
\text { fluorescens }\end{array}$ & 1.00 & $\begin{array}{c}23.73 \\
(29.15)\end{array}$ & $\begin{array}{c}42.13 \\
(40.47)\end{array}$ & $\begin{array}{c}76.27 \\
(60.85)\end{array}$ & $\begin{array}{c}47.38 \\
(43.50)\end{array}$ & $\begin{array}{c}24.80 \\
(29.87)\end{array}$ & $\begin{array}{c}42.67 \\
(40.78)\end{array}$ & $\begin{array}{c}77.07 \\
(61.39)\end{array}$ & $\begin{array}{c}48.18 \\
(43.96)\end{array}$ & 47.78 & 1.56 \\
\hline Salicylic Acid & 0.25 & $\begin{array}{c}13.87 \\
(21.86)\end{array}$ & $\begin{array}{c}33.60 \\
(35.43)\end{array}$ & $\begin{array}{c}57.33 \\
(49.22)\end{array}$ & $\begin{array}{c}34.93 \\
(36.23)\end{array}$ & $\begin{array}{c}11.20 \\
(19.55)\end{array}$ & $\begin{array}{c}28.80 \\
(32.46)\end{array}$ & $\begin{array}{c}54.67 \\
(47.68)\end{array}$ & $\begin{array}{c}31.56 \\
(34.18)\end{array}$ & 33.24 & 31.50 \\
\hline Borax & 0.50 & $\begin{array}{c}16.27 \\
(23.79)\end{array}$ & $\begin{array}{c}36.00 \\
(36.87)\end{array}$ & $\begin{array}{c}69.07 \\
(56.21)\end{array}$ & $\begin{array}{c}40.44 \\
(39.49)\end{array}$ & $\begin{array}{c}16.80 \\
(24.20)\end{array}$ & $\begin{array}{c}33.87 \\
(35.59)\end{array}$ & $\begin{array}{c}63.73 \\
(52.97)\end{array}$ & $\begin{array}{c}38.13 \\
(38.14)\end{array}$ & 39.29 & 19.05 \\
\hline $\mathrm{K}_{2} \mathrm{SO}_{4}$ & 1.00 & $\begin{array}{c}18.13 \\
(25.20)\end{array}$ & $\begin{array}{c}37.60 \\
(37.82)\end{array}$ & $\begin{array}{c}71.20 \\
(57.54)\end{array}$ & $\begin{array}{c}42.31 \\
(40.58)\end{array}$ & $\begin{array}{c}18.93 \\
(25.79)\end{array}$ & $\begin{array}{c}35.20 \\
(36.39)\end{array}$ & $\begin{array}{c}67.73 \\
(55.39)\end{array}$ & $\begin{array}{c}40.62 \\
(39.59)\end{array}$ & 41.47 & 14.56 \\
\hline $\mathrm{CaSO}_{4}$ & 1.00 & $\begin{array}{c}15.20 \\
(22.95)\end{array}$ & $\begin{array}{c}35.20 \\
(36.39)\end{array}$ & $\begin{array}{c}65.60 \\
(54.09)\end{array}$ & $\begin{array}{c}38.67 \\
(38.45)\end{array}$ & $\begin{array}{c}15.20 \\
(22.95)\end{array}$ & $\begin{array}{c}31.20 \\
(33.96)\end{array}$ & $\begin{array}{c}58.93 \\
(50.15)\end{array}$ & $\begin{array}{c}35.11 \\
(36.34)\end{array}$ & 36.89 & 23.99 \\
\hline $\begin{array}{l}\text { Metalaxyl } \\
0.1 \%\end{array}$ & 0.10 & $\begin{array}{c}12.80 \\
(20.96)\end{array}$ & $\begin{array}{c}29.60 \\
(32.96)\end{array}$ & $\begin{array}{c}52.00 \\
(46.15)\end{array}$ & $\begin{array}{c}31.47 \\
(34.12)\end{array}$ & $\begin{array}{c}9.60 \\
(18.05)\end{array}$ & $\begin{array}{c}23.47 \\
(28.97)\end{array}$ & $\begin{array}{c}46.93 \\
(43.24)\end{array}$ & $\begin{array}{c}26.67 \\
(31.09)\end{array}$ & 29.07 & 40.11 \\
\hline $\begin{array}{c}\text { Metalaxyl } \\
0.2 \%\end{array}$ & 0.20 & $\begin{array}{c}12.53 \\
(20.73)\end{array}$ & $\begin{array}{c}29.33 \\
(32.79)\end{array}$ & $\begin{array}{c}51.73 \\
(45.99)\end{array}$ & $\begin{array}{c}31.20 \\
(33.96)\end{array}$ & $\begin{array}{c}9.33 \\
(17.79)\end{array}$ & $\begin{array}{c}23.20 \\
(28.79)\end{array}$ & $\begin{array}{c}46.67 \\
(43.09)\end{array}$ & $\begin{array}{c}26.40 \\
(30.92)\end{array}$ & 28.80 & 40.66 \\
\hline $\begin{array}{c}\text { Metalaxyl } \\
0.3 \%\end{array}$ & 0.30 & $\begin{array}{c}12.27 \\
(20.50)\end{array}$ & $\begin{array}{c}29.07 \\
(32.62)\end{array}$ & $\begin{array}{c}51.47 \\
(45.84)\end{array}$ & $\begin{array}{c}30.93 \\
(33.79)\end{array}$ & $\begin{array}{c}9.07 \\
(17.52)\end{array}$ & $\begin{array}{c}22.93 \\
(28.61)\end{array}$ & $\begin{array}{c}46.40 \\
(42.94)\end{array}$ & $\begin{array}{c}26.13 \\
(30.74)\end{array}$ & 28.53 & 41.21 \\
\hline KCl & 1.00 & $\begin{array}{c}20.00 \\
(26.57)\end{array}$ & $\begin{array}{c}39.20 \\
(38.76)\end{array}$ & $\begin{array}{c}72.00 \\
(58.05)\end{array}$ & $\begin{array}{c}43.73 \\
(41.40)\end{array}$ & $\begin{array}{c}20.00 \\
(26.57)\end{array}$ & $\begin{array}{c}37.33 \\
(37.66)\end{array}$ & $\begin{array}{c}71.47 \\
(57.71)\end{array}$ & $\begin{array}{c}42.93 \\
(40.94)\end{array}$ & 43.33 & 10.71 \\
\hline $\mathrm{ZnSO}_{4}$ & 0.50 & $\begin{array}{c}21.33 \\
(27.51)\end{array}$ & $\begin{array}{c}41.07 \\
(39.85)\end{array}$ & $\begin{array}{c}73.87 \\
(59.26)\end{array}$ & $\begin{array}{c}45.42 \\
(42.37)\end{array}$ & $\begin{array}{c}22.40 \\
(28.25)\end{array}$ & $\begin{array}{c}39.20 \\
(38.76)\end{array}$ & $\begin{array}{c}75.47 \\
(60.31)\end{array}$ & $\begin{array}{c}45.69 \\
(42.53)\end{array}$ & 45.56 & 6.14 \\
\hline Check & - & $\begin{array}{c}24.80 \\
(29.87)\end{array}$ & $\begin{array}{c}43.20 \\
(41.09)\end{array}$ & $\begin{array}{c}76.80 \\
(61.21)\end{array}$ & $\begin{array}{c}48.27 \\
(44.01)\end{array}$ & $\begin{array}{c}25.33 \\
(30.22)\end{array}$ & $\begin{array}{c}43.47 \\
(41.25)\end{array}$ & $\begin{array}{c}77.60 \\
(61.75)\end{array}$ & $\begin{array}{c}48.80 \\
(44.31)\end{array}$ & 48.53 & 0.00 \\
\hline SEm \pm & - & 0.48 & 0.86 & 0.65 & - & 0.73 & 0.86 & 1.70 & - & - & - \\
\hline CD 5\% & - & 1.39 & 2.47 & 1.87 & - & 2.10 & 2.48 & 4.89 & - & - & - \\
\hline
\end{tabular}

Values in parenthesis are angular transformed values

DAS= Days after sowing 
The per cent disease index in cv. RH-749 was recorded at 70, 80 and 90 days after sowing of the mustard. All the treatments were found significantly better over the check (Table 3). Among all the treatments Metalaxyl 0.3\% found superior which reduce 41.21 per cent disease in comparison with the control. However, the different concentrations of Metalaxyl were found at par with each other at 70, 80 and 90 days after sowing during 2015-16 and 2016-17. In bio agents, Both Trichoderma viride and Pseudomonas fluorescens were found non-significant at 70, 80 and 90 days after sowing during 2015-16 and 2016-17 in comparison with the control. Among abiotic agents, salicylic acid at $0.25 \%$ reduce disease significantly in 2015-16 and 2016-17. salicylic acid $0.25 \%$ reduce $31.50 \%$ white rust disease over the control followed by calcium sulphate at $1.00 \%$ which reduce disease $23.99 \%$. Zinc sulphate at $0.50 \%$ was found least effective abiotic chemical which reduce $6.14 \%$ disease (Table 4 ).

Tirmali and Kolte (2012) reported the efficacy of several plant defense activators in the management of Albugo candida pathogen during 2003-04. They used varuna cultivar to study induction of host resistance in mustard against the pathogen. They found calcium sulphate, potassium chloride, potassium sulphate, zinc sulphate and borax significantly superior effective in reduction the pustules size of white rust on the mustard leaves with comparison to control.

Sharma and Kolte (1994) reported that potassium fertilized plants exhibited 30 to 45 per cent less disease severity of Alternaria blight based on the number and size of the spots, average disease index on leaf and pods. Tewari (1991) found that foliar application of the calcium reduce the per cent disease severity of Alternaria blight in rapeseed. Antonova et al., (1984) and Dixon et al., (1987) reported that boron application in the cabbage increase resistance to club root.

\section{References}

Antonova, T.S., Zaichlor, V.E. and Kalinchenlw, T.V. 1984. Role of calcium in the resistance of the sunflower anthodium to gray root. Sci skokhozyaistvernnaya Biologiya, 11: 67-68.

Becot, S., Pajot, E., Corre, D., Monot, C. and Silne, D. 2000. Phytogard $\left(\mathrm{K}_{2} \mathrm{PHO}_{3}\right)$ induces localized resistance in cauliflower to downy mildew of crucifers. Crop Protection, 19: 417-425.

Benhamon, N. and Garand, C. 2001. Cytological analysis of defense related mechanisms in pea root tissues in response to colonization by nonpathogenic Fusarium oxysporum Fo47. Phytopathology, 91: 730-740.

Biswas, C., Singh, R. and Kumar, P. V. 2011. Dynamics of white rust disease in mustard (Brassica juncea) in relation to date of sowing and weather parameters. Indian Journal of Agricultural Sciences, 81(12): 1187-1190.

Cohen, Y. 1994. 3-Amminobutric acid induces systemic resistance against Peronospora tabacina, Physiol Mol Plant Pathol., 44: 273-288.

De Oliveira, D. E., Seurinck, J., Inze, D., Van Montague, M. and Botterman, J. 1990. Differential expression of five Arobidopsis genes encoding glycinerice protein. The Plant cell, 2: 427-436.

Dixon, G. R., Naiki, T., Webster, A. and Wilson, F. 1987. Integrated use of boron calcium cynamide and nitrogen for control of club root (Plasmodiophora brassicae), In Crop protection in Northern Britan, 399-404.

Droly, S., Vinokur, V., Wiess, B., Cohen, L., Daws, A., Goldschmidt, E. E. and Porat, R. 2002. Induction of resistance to Pennicilium digitatum in grape fruit by the yeast bio-control agent Candida oleophila. Phytopathol., 92: 93-399.

Durrant, W. E. and Dong, X. 2004. Systemic 
acquired resistance. Annu. Rev. Phytopathol., 42: 185-209.

Gorlach, J., Volrath, S., Oostendorp, M., Kogel, K. H., Beckhove, U., Staub, T., Ward, E., Kessmann, H. and Ryals, J. 1996. Benzothiadiazole, a novel class of inducers of systemic acquired resistance, activates systemic resistance in wheat. Plant Cell, 8: 629-643.

Graham, J. H. and Leite, R. P. 2004. Lack of control of citrus canker by induced systemic resistance compounds. Plants Dis., 88: 745-750.

Hammerschmidt, R. 1999. Induced disease resistance: how do induced plants stop pathogens. Physiol. Mol. Plant Pathol., 55: $77-84$.

Holopainen, J. K. Heijari, J., Nerg, A. M., Vuorinen, M. and Kainulainen, P. 2009. Potencial for the use of exogenous chemical elicitors in disease and insect pest management of conifer seedling production. Open. For. Sci. J., 2:17-24.

Howell, C. R., Hanson, L. E., Spitanovic, R. D. and Puckhaber, C. S. 2000. Induction of terpenoid synthesis in cotton roots and control of Rhizoctonia solani by seed treatments with $T$. virens. Phytopathol., 90: 248-252.

Kaur, A. and Kolte, S. J. 2001. Protection of mustard plants against staghead phase of white rust by Foliave treatment with benzothiadiazole-An activator of plant defense system. J Mycol Pl Pathol., 31: 133-138.

Kobayashi, A., Tai, A., Hiroshi, K. and Kazuyoshi, K. 1993. Elicitor-active oligosaccharides from algal laminarin stimulate the production of antifungal compounds in alfalfa. Z. Naturforsch., 48: 575-579.

Liu, L., Kloepper, J. W. and Tuzun, S. 1995. Induced systemic resistance in cucumber against bacterial angular spot by plant-growth promoting rhizobacteria. Phytopathology, 85: 843-
847.

Macmillan, R. T., Graves, W. and Mc Laughlin, H. J. 2000. Key Plex effect on Sigatoka of banana. PWC Florida State Hort Soc 112: 192-193.

Matheron, M. E. and Porchas, M. 2002. Suppression of Phytophthora root and crown rot on pepper plants treated with Acibenzolar-SMethyl. Plant Dis., 86:292-297.

Mathur, P., Sharma, E., Singh, S. D. Bhatnagar, A. K., Singh, V. P. and Kapoor, R. 2013. Effect of elevated $\mathrm{CO}_{2}$ on infection of three foliar diseases in oilseed Brassica juncea. Journal of Plant Pathology, 95(1): 135-144.

Narusaka, X., Naraska, M., Horio, T. and Ishii, H. 1999. Comparison of local and systemic induced resistance in cucumber plants treated with benzothiadiazoles or salicylic acid. Plant Cell Physiol., 40:388-395.

Omidi, H. 2010. Changes of proline content and activity of antioxidative enzymes in two canola genotype under drought stress. Am. J. Plant Physiol., 5:338-349.

Ozeretskovskaya, O. L. and Vasyukova, N. I. 2002. The use of elicitors for protection of cultured plants demands caution. Appl. Biochem. Microbiol., 38:277-279.

Pajot, E., Corre, D. Le. and Silue, D. 2001. Phytogard and DL-â-amonobutynic acid (BABA) induced resistance to downy mildews (Bremia lactucae) in lettuce (Lettuce sativa). Eur J Plant Pathol., 107: 861-864.

Panse, V.G. and Sukhatme, P.V. 1985. Statistical Methods for Agricultural Workers, 4th ed., ICAR, New Delhi, 347 p.p

Romero, A. M., Kousik, C. C. and Ritchie, D. F. 2001. Resistance to bacterial spot in bell pepper induced by acibenzolar-5methyl. Plant Dis., 85: 189-194.

Ryals, J., Uknes, S. and Ward, E. 1994. Systemic acquired resistance. Plant 
Physiol., 104(4): 1109-1112.

Safari, S., Soleimani, M. J., Mohajer, A. and Fazlikhani, L. 2013. Possible structureactivity profile of salicylate derivatives: their relationship on induction of systemic acquired resistance. Journal of Agricultural Technology, 9(5): 12151225.

Sharma, S. R. and Kolte, S. J. 1994. Effect of soil applied NPK fertilizers on severity of black spot disease (Aternaria brassicae) and yield of oil seed rape. Plant and soil, 167:313-320.

Singh, U.S., Dought, K. J., Nashaat, N. I., Bennet, R. N. and Kolte, S. J. 1999. Induction of systemic resistance to Albugo candida in Brassica juncea by pre or co-inoculation with an incompatible isolate. Phytopathol., 89: 1226-1232.

Spletzer, M. E. and Enyedi, A. J. 1999. Salicylic acid induces resistance to Alternaria solani in hydroponically grown tomato. Phytopathology, 89(9): $722-727$

Surekha, C. H., Neelapu, N. N. R., Siva Prasad, B. and Sankar G. P. 2014. Induction of defense enzymes and phenolic content by Trichoderma viride in Vigna mungo infested with Fusarium oxysporum and Alternaria alternate. International Journal of Agricultural Science and Research, 4(4): 31-40.

Tewari, J. P. 1991. Current understanding of resistance to Alternaria brassicae in crucifers. Proc. GCRlC, $8^{\text {th }}$
International. Rapeseed Congress. July 9- 11, Saskatoon, Canada, 2: 471-476.

Tirmali, A. M. and Kolte, S. J. 2012. Induction of host resistance in mustard with non- conventional chemicals against white rust (Albugo candida). $j$. pl. dis. sci., 7(1): 27-31.

Verhagen, B. W. M., Glazebrook, J. T., Chang, H. S. and Van Loon, L. C. 2004. The transcriptome of Rhizobacteria induced Systemic resistance in Arabidopsis. Mol Plant- Microbe Interact., 17: 985-908.

Vishwanath, C. Kolte, S. J., Singh, M. P. and Awasthi, R. P. 1999. Introduction of resistance in mustard (B. juncea) against Alternaria black spot with an avirulent Alternaria brassicae isolate. D. Euro J Plant Pathol., 105: 217-220.

Wei, C., Laby, R. J., Zumoff, C. H., Bauer, H. W., He, S. Y., Colmer, A. and Beer, S. V. 1992. Harpin, elictor of the hypersensitive response produced by the plant pathogen Erwinia amylovora. Science, 257:1-132.

Wheeler, B.E.J. 1969. An Introduction to Plant Diseases. John Wiley and Sons, London, UK.

Yalpani, N. and Raskin, I. 1993. Salicylic acid: a systemic signal in induced plant resistance. Trends Microbiol., 1:88-92.

Zhao, J., Davis, L. C. and Verporte, R. 2005. Elicitor signal transduction leading to production of plant secondary metabolites. Biotechnol. Adv., 23: 283333.

\section{How to cite this article:}

Hanuman Singh, R.S. Ratnoo, A. Trivedi, H.K. Jain, V. Saharan and Sharma, F.L. 2019. Effect of Plant Defense Activators on White Rust of Mustard CV. RH- 749 under Artificial Epiphytotic Conditions. Int.J.Curr.Microbiol.App.Sci. 8(10): 1113-1122. doi: https://doi.org/10.20546/ijcmas.2019.810.130 